\title{
ANALOGÍAS A PRIORI Y APLICACIÓN DE CONCEPTOS
}

\section{A PRIORI ANALOGIES AND APPLICATION OF CONCEPTS}

\author{
Fabián Bernache Maldonado \\ Profesor Investigador Titular \\ Departamento de Filosofía \\ Universidad de Guadalajara, Centro Universitario de Ciencias Sociales y Humanidades. \\ Calle Guanajuato No. 1045, Colonia Alcalde Barranquitas, C.P. 44260. \\ Guadalajara, Jalisco, México. \\ Teléfono: +523338193377 \\ fabian.bernachelaacademicos.udg.mx
}

\begin{abstract}
¿Constituyen las analogías a priori un tipo de argumento distinto de los argumentos deductivos? Mi objetivo en este artículo es abordar esta larga polémica. Basándome en una tesis general acerca de la relación entre nuestra capacidad de aplicar conceptos y nuestra capacidad intuitiva de argumentar lógicamente, sostengo que los principios de fundamentación racional que empleamos en las analogías a priori son esencialmente los mismos que los que empleamos en la argumentación deductiva. Por consiguiente, al menos desde un punto de vista lógico, no hay razón para admitir que las analogías a priori constituyan un tipo distinto de argumento. No por ello debemos suponer, sin embargo, que el uso de analogías a priori es prescindible.
\end{abstract}

Palabras clave: argumentos, analogías, juicios, razones, capacidades. 


\section{8 - Cogency, Journal of reasoning and argumentation}

Do a priori analogies constitute a type of argument distinct from deductive arguments? My aim in this paper is to address this long controversy. I claim that the principles of rational foundation that we use in a priori analogies are essentially the same as those that we use in deductive argumentation. This view is grounded on a general thesis about the relation between our capacity to apply concepts and our intuitive capacity to logically argue. Therefore, at least from a logical point of view, there is no reason to admit that a priori analogies constitute a distinct type of argument. We should not suppose, however, that the use of a priori analogies is dispensable

Keywords: arguments, analogies, judgments, reasons, capacities. 


\section{Introducción}

El uso de analogías en los argumentos es uno de los fenómenos más complejos que intentan explicar los teóricos de la argumentación. Una de las principales interrogantes al respecto es si los argumentos analógicos constituyen un tipo de argumento distinto de los demás, esto es, distinto de los argumentos deductivos, inductivos o abductivos. Si se admite que los argumentos analógicos son de un tipo distinto de los otros, ¿cómo exactamente difieren de ellos?, ¿en virtud de qué principios respaldan su conclusión?, ¿su uso es igualmente racional que el uso de argumentos deductivos, inductivos o abductivos? Si se admite, en cambio, que los argumentos analógicos no constituyen un tipo distinto de los demás, ¿cómo explicar el uso frecuente de analogías en la argumentación?, ¿cuál es su función?, ¿es posible prescindir de los argumentos analógicos? ${ }^{1}$

Mi objetivo en este artículo es posicionarme en este debate enfocándome, sobre todo, en los argumentos analógicos que han sido designados con la expresión "analogías a priori". La noción de analogía a priori fue introducida por Trudy Govier (1989) para referir a aquellos argumentos analógicos que respaldan su conclusión de manera definitiva y que pueden utilizar como elemento análogo situaciones o casos que no son reales (una caracterización más detallada será presentada en la sección I). Es en torno a la noción de analogía a priori que el debate se ha ido construyendo, aunque no todos los autores han estado de acuerdo con esta terminología. ${ }^{2}$ La discusión sobre la naturaleza de las analogías a priori y, en particular, sobre la cuestión de si las analogías a priori constituyen un tipo de argumento distinto de los argumentos deductivos, comienza con la fructífera polémica entre Govier $(1989,2002)$ y Waller (2001). En las secciones I y II analizaré la postura de estos autores con el fin, esencialmente, de precisar la problemática y poner énfasis en aquellos puntos que me permitirán introducir mi propia propuesta.

En la sección III presentaré una tesis general acerca de lo que llamo "nuestra capacidad intuitiva de argumentar lógicamente", es decir nuestra capacidad intuitiva de identificar y formular buenas razones para respaldar o refutar aserciones en un intercambio argumentativo. De acuerdo con esta tesis general, dicha capacidad está fundamentalmente basada en nuestra sensibilidad a las condiciones de aplicación de nuestros conceptos. Apoyándome en las ideas que ahí discutiré, en la sección IV abordaré la cuestión de la naturaleza de las analogías a priori. La posición que defiendo es que los principios de fundamentación racional

1 Entre los teóricos que sostienen que los argumentos analógicos (especialmente las analogías a priori) constituyen un tipo de argumento distinto de los otros están Alvargonzález (2020), Bermejo-Luque (2012, 2014), Freeman (2013), Govier (1989, 2002, 2014), Guarini (2004), Juthe (2005, 2015, 2019), Van Eemeren y Garssen (2014) y Van Eemeren y Snoek Henkemans (2017), mientras que entre aquellos que sostienen lo contrario están Shecaira (2013) y Waller (2001).

2 Véase, por ejemplo, Bermejo-Luque (2014). 
que empleamos en las analogías a priori son esencialmente los mismos que los que empleamos en la argumentación deductiva. Tanto en el caso de un argumento deductivo, como en el caso de una analogía a priori, respaldamos la conclusión de nuestro argumento basándonos en conocimiento que describo como "conocimiento constitutivo de la capacidad de aplicar conceptos". La conclusión que pretendo extraer de este planteamiento - que será desarrollado detalladamente - es que, al menos desde un punto de vista lógico, las analogías a priori no constituyen un tipo de argumento distinto de los argumentos deductivos. No por ello debemos suponer, sin embargo, que el uso de estas analogías en nuestras prácticas argumentativas es prescindible.

\section{El planteamiento de Govier}

De acuerdo con Trudy Govier (1989), los argumentos analógicos no son asimilables a ningún otro tipo de argumento, sino que constituyen un tipo distinto por derecho propio. Govier señala también que, aunque los argumentos analógicos no son inductivos ni deductivos, es necesario distinguir entre analogías inductivas y analogías a priori (Govier, $1989,2014)$ Una característica distintiva de una analogía inductiva es la utilización como elemento análogo de un caso real cuyas propiedades relevantes son constatadas empíricamente. Una analogía a priori, en cambio, puede utilizar como elemento análogo un caso hipotético o, incluso, uno fantasioso o muy poco probable (Govier, 1989). Otra diferencia importante es que, mientras que la conclusión de una analogía inductiva es predictiva y establecida de manera probable, una analogía a priori establece de forma definitiva una conclusión que no es predictiva, sino que trata de la manera en que debe ser caracterizado el elemento meta, es decir aquel que es comparado con el elemento análogo y sobre el cual se argumenta (Govier, 1989). ${ }^{3}$

Un ejemplo de Govier para ilustrar las analogías inductivas es el de la posibilidad de abolir la guerra en el mundo mediante la acción civil y la promulgación de reformas. Govier señala que algunas personas han argumentado a favor de esta posibilidad haciendo una analogía entre la abolición de la guerra y la abolición de la esclavitud. Quienes utilizan este argumento pretenden establecer, de manera probable, la conclusión predictiva de que la abolición de la guerra se logrará, como ha sido lograda la de la esclavitud, mediante la acción civil y la promulgación de reformas.

3 Bermejo-Luque (2014) utiliza los términos "analogía cuantitativa" y "analogía cualitativa" para establecer básicamente esta misma distinción, mientras que Van Eemeren y Garssen (2014) utilizan los términos "analogía descriptiva" y "analogía normativa". 
Para ilustrar las analogías a priori, Govier presenta varios ejemplos, entre ellos un argumento de Locke (1689/2006, p. 173):

Que el agresor que se pone en un estado de guerra con otro e injustamente invade sus derechos no puede jamás, como resultado de esa guerra injusta, tener derecho alguno sobre el conquistado es algo en lo que estará de acuerdo todo hombre que no piense que los ladrones y piratas tienen derecho a mandar sobre aquellos a quienes han dominado por la fuerza; y tampoco podrá estar nadie obligado a cumplir promesas que le han sido arrancadas ilegalmente mediante intimidación y violencia. Si un ladrón entrara en mi casa y, poniéndome una daga en el cuello, me obligase a firmar un documento en el que se dijera que yo estoy cediendo mi propiedad, ¿le daría esto algún derecho para apropiársela? Exactamente el mismo derecho tendrá para someterme un conquistador que me obligase a ello por la fuerza de las armas. La injuria y el crimen serían idénticos, tanto si fuesen cometidos por quien lleva en la cabeza una corona como si fuesen perpetrados por un vulgar delincuente.

En este argumento, Locke compara el derecho de un conquistador a mandar sobre un conquistado a quien ha sometido de forma violenta con el derecho que tendrían ladrones y piratas a mandar sobre aquellos a quienes han igualmente sometido por la fuerza. Puesto que, al menos desde la perspectiva de Locke, las situaciones son análogas, si rechazamos el derecho de los segundos y lo consideramos ilegítimo, tenemos que hacer lo mismo con el derecho del primero. El caso de los piratas es, desde luego, un caso real. En cambio, el caso del ladrón que somete a su víctima con una daga es más bien hipotético o, al menos, no es un caso que hubiéramos tenido que constatar empíricamente para que fuera posible extraer la conclusión del argumento. Es fundamentalmente esta característica que lleva a Govier a considerar que una forma adecuada de designar este tipo de argumentos analógicos es utilizando la expresión "analogías a priori”. Notemos, además, que la conclusión del argumento de Locke no es predictiva, sino que trata de la manera en que debe ser considerado el derecho de un conquistador a mandar sobre un conquistado a quien ha sometido violentamente. Como ya fue mencionado, según el argumento, tal derecho es ilegítimo. Finalmente, la conclusión es establecida de forma definitiva: si el derecho de ladrones y piratas es ilegítimo, el del conquistador lo es también, y esto en virtud de la analogía entre ambas situaciones.

En este artículo, como ya fue señalado, me concentraré en las analogías a priori y, en esta sección, expondré las razones por las cuales Govier considera que es un error asimilarlas a argumentos deductivos. Primeramente, Govier observa que una manera de reconstruir la estructura de las analogías a priori es añadiendo una aserción que no suele figurar explícitamente en estos argumentos, pero que parece estar presupuesta en ellos. Se trata de la aserción 4': 
12 - Cogency, Journal of reasoning and argumentation

1. A posee las características $x, y, z$.

2. B posee las características $x, y, z$.

3. A es $w$.

4. Es en virtud de $x, y, z$ que $A$ es $w$.

5. Por consiguiente, $B$ es $w$.

De la aserción 4’ no es difícil pasar, nos dice Govier, a la aserción universal 4*:

$4^{\star}$. Todo aquello que posee las características $x, y, z$ es $w$.

Las dificultades surgen cuando asumimos que una aserción como $4^{\star}$ constituye una premisa implícita de las analogías a priori y reconstruimos la estructura de estos argumentos de la siguiente manera:

1. A posee las características $x, y, z$.

2. B posee las características $x, y, z$.

3. A es $w$.

$4^{*}$. Todo aquello que posee las características $x, y, z$ es $w$.

5. Por consiguiente, $B$ es $w$.

En esta reconstrucción, el problema es evidente: 1 y 3 no cumplen función alguna en el argumento. Dado que 1 y 3 son las premisas que introducen el elemento análogo e indican sus propiedades relevantes, si el análisis nos conduce a un tal resultado, es razonable suponer que las analogías a priori son asimilables a argumentos deductivos. En el caso de las analogías inductivas, una reducción a argumentos inductivos convencionales sería también posible, señala Govier, si introducimos en la reconstrucción de su estructura una premisa similar a $4^{*}$ que, en vez de ser un enunciado universal, sería una aserción como: "generalmente, aquello que posee las características $x, y, z$ es $w$ ".

¿Por qué piensa Govier que es un error reducir las analogías a priori a argumentos deductivos? Tal reducción conlleva una dificultad importante: si las analogías que figuran en estos argumentos son superfluas, ¿por qué las utilizamos? Para Govier, sin embargo, el hecho de que la reconstrucción deductivista de las analogías a priori relegue dos de sus premisas a la categoría de información redundante, si bien es problemático, no constituye una dificultad decisiva. Encontrar una función convincente para 1 y 3 no sería una tarea 
imposible. Según Govier, no es absurdo asumir que $4^{\star}$ expresa información presupuesta en toda analogía a priori. En efecto, si admitimos que $A$ y $B$ comparten las propiedades $x, y, z \mathrm{e}$ igualmente que es en virtud de $x, y, z$ que $A$ es $w$, y si inferimos de estas premisas que $B$ es $w$, ¿no estamos ya implícitamente asumiendo que todo lo que es $x, y, z$ es $w$ ? ¿Por qué entonces no admitir que $4^{*}$ es una premisa implícita de las analogías a priori y, por consiguiente, que estos argumentos analógicos son asimilables a argumentos deductivos?

Para abordar esta cuestión, Govier comienza distinguiendo dos formas de presuponer información en un argumento: como información de trasfondo y como premisas implícitas (Govier, 1989). Por ejemplo, de acuerdo con Govier, los argumentos deductivos presuponen, como información de trasfondo, el principio de no contradicción, pero este principio no constituye una premisa implícita de los argumentos deductivos. La información de trasfondo que un argumento presupone es siempre distinta de la información que aportan sus premisas (explícitas o implícitas). Dada esta distinción, es posible admitir que $4^{\star}$ expresa información presupuesta en las analogías a priori sin tener que asumir que dicha aserción constituye una premisa implícita de este tipo de argumentos. Sin embargo, Govier rechaza tanto la idea de que $4^{\star}$ constituya una premisa implícita como la idea de que $4^{\star}$ exprese información de trasfondo, pues considera que, con estas propuestas, se asume erróneamente que $4^{\star}$ posee cierta forma de prioridad lógica o epistémica. Para Govier, existe una tercera posibilidad: el enunciado universal $4^{\star}$ es implicado por las analogías a priori. Así, Govier señala que una persona que emplea una analogía a priori se compromete con $4^{\star}$, no porque este enunciado esté presupuesto o sea una premisa implícita del argumento, sino porque, si lo que dice la persona en su argumento es correcto, un tal enunciado tiene que ser verdadero. ${ }^{4}$

El esquema deductivista con el que se ha pretendido reconstruir la estructura de las analogías a priori puede hacernos creer, nos dice Govier, que es claro para aquellos que emplean estos argumentos cuáles son las propiedades que $A$ y $B$ comparten y en virtud de las cuales se sigue la conclusión. Sin embargo, de acuerdo con Govier, una persona puede perfectamente percibir la similitud entre $A$ y $B$ y comprender el hecho de que, en virtud de esta similitud, cierta propiedad $w$ que $A$ posee puede igualmente ser atribuida a $B$, sin que por ello la persona haya podido identificar las propiedades exactas que explican que $A$ y $B$ sean similares, ni podido captar con claridad la relación que existe entre la posesión de estas propiedades y la posesión de la propiedad $w$. Según Govier, esto es lo que suele

4 Utilizando el término "aserción-U" para designar el enunciado universal implicado por las analogías a priori les decir $4^{*}$ ), Govier intenta explicar su uso del término "implicado" señalando lo siguiente: "El uso de un argumento por analogía compromete a la argumentadora con una aserción- $U$ en el sentido de que si lo que dice en su argumento es correcto, entonces una aserción-U debe ser verdadera" (Govier, 1989, p.148). Esta cita textual ha sido traducida por mí, así como el resto de las citas textuales de obras en inglés que aparecen en este artículo. 
suceder cuando empleamos analogías a priori. Tal situación sugiere, de acuerdo con nuestra autora, que el enunciado universal presupuesto en las analogías a priori no es una premisa implícita, ni tampoco información de trasfondo.

Govier hace igualmente hincapié en el hecho de que, para evaluar una analogía a priori, no es necesario conocer el contenido específico del enunciado universal implicado por ella, sino que basta con podamos señalar diferencias importantes entre $A$ y $B$ y mostrar cómo esas diferencias afectan el respaldo que, pretendidamente, las premisas dan a la conclusión. Así, el enunciado universal en cuestión no solamente puede ser difícil de formular, sino que insertarlo como una premisa implícita no sería ni necesario ni útil (Govier, 1989). Para Govier, esto muestra que el esquema deductivista de las analogías a priori es incorrecto. De ahí que debamos reconocer que estas analogías constituyen un tipo de argumento distinto de los argumentos deductivos.

Sin embargo, no es del todo claro que la explicación que da Govier de su uso del término "implicado" permita afirmar que la autora ha logrado en efecto identificar una tercera posibilidad, distinta de las otras dos. Tanto si admitiéramos que $4^{*}$ expresa información de trasfondo como si admitiéramos que se trata de una premisa implícita, podríamos también decir que, si la analogía a priori es correcta, $4^{\star}$ debe ser verdadero. Como fue señalado, con la introducción de una tercera posibilidad, Govier trata de evitar que se otorgue una prioridad lógica o epistémica a $4^{\star}$ que, según ella, este enunciado no tiene. Pero, mientras Govier no logre mostrar con más claridad en qué sentido su uso del término "implicado" constituye realmente una tercera posibilidad, no es posible admitir que haya podido evitar este supuesto problema.

En la sección III, intentaré mostrar que, efectivamente, existe una tercera posibilidad, es decir una tercera forma de presuponer información en un argumento que es distinta a la vez de la información de trasfondo y de las premisas implícitas. Se trata del conocimiento implícito que constituye nuestras capacidades conceptuales. Sin embargo, en la sección IV, argumentaré que, a partir del reconocimiento de esta forma de presuponer información, es posible concluir que las analogías a priori no son argumentos distintos de los argumentos deductivos. Pero antes, presentaré la importante respuesta que da Waller al planteamiento de Govier.

\section{La réplica de Waller}

En contraste con Govier, Bruce N. Waller (2001) afirma que la reconstrucción deductivista de las analogías a priori permite una mejor comprensión de su funcionamiento, en la medida en que, precisamente, tal reconstrucción admite como parte integral de su estructura el principio universal que en ellas se presupone. De acuerdo con Waller, los argumentos analógicos no constituyen un tipo distinto de los otros y las analogías a priori, 
en particular, son esencialmente argumentos deductivos. Para representar la estructura de las analogías a priori, Waller (2001) propone el siguiente esquema (que presentamos con ligeras modificaciones):

1. Ambos admitimos que $A$ es $w$.

2. La razón más plausible para creer que $A$ es $w$ es la aceptación del principio $P$.

3. $P$ implica que $B$ es $w$ (el hecho de que $B$ es $w$ es conforme con $P$ )

4. Luego, si somos congruentes, debemos admitir que $B$ es $w$.

El principio $P$ no es otra cosa que el enunciado universal que, tanto para Waller como para Govier, toda analogía a priori presupone (o “implica”, en el caso de Govier). Nuestros autores concuerdan también en el hecho de que, en muchos casos, el contenido de este enunciado no es claramente conocido por la persona misma que formula el argumento. Sin embargo, desde la perspectiva de Waller, esto no es una razón para negar que $P$ sea un componente del argumento, pues él afirma que una de las funciones centrales de las analogías a priori es hacernos reflexionar acerca de los principios que implícitamente aceptamos y sus implicaciones (Waller, 2001).

Consideremos de nuevo la analogía a priori de Locke. Es claro que Locke supone, en su argumentación, que su lector está de acuerdo con él en el hecho de que los ladrones y piratas no tienen derecho a mandar sobre aquellos a quienes han sometido violentamente. En términos del esquema de Waller, Locke supone que ambos, su lector y él, admiten que $A$ es $w$. Según Waller, la aceptación del hecho de que $A$ es $w$ presupone la aceptación de un principio universal $P$, pues es precisamente de un tal principio que derivaría la aceptación del hecho de que $A$ es $w$. Si bien es difícil negar que un principio de este tipo sea implícitamente aceptado en el argumento de Locke, es preciso también reconocer que, incluso para las personas que aceptan el argumento, no necesariamente es claro cuál sería su formulación correcta. A pesar de ello, para Waller, el argumento presupone un tal principio como una de sus partes integrales y nos está forzando a pensar en él y en sus implicaciones (Waller, 2001). En este ejemplo, el argumento nos estaría forzando a pensar si el principio en virtud del cual rechazamos que ladrones y piratas tengan derecho a mandar sobre aquellos a quienes han sometido por la fuerza es también aplicable al caso de un gobernante que somete violentamente a otro. Y la respuesta, al menos según el argumento, es que sí.

De acuerdo con Waller, el papel que juega el principio universal $P$ en una analogía $a$ priori puede apreciarse más claramente si imaginamos un diálogo con una persona que re- 
chaza un argumento de este tipo. A alguien que no quisiera aceptar el argumento de Locke podríamos preguntarle: "Estás de acuerdo conmigo en el hecho de que los ladrones y piratas no tienen derecho a mandar sobre aquellos a quienes han sometido violentamente. ¿Por qué piensas que no lo tienen?" Según Waller, al plantear esta pregunta, estaríamos forzando a nuestro interlocutor a identificar y formular el principio universal $P$. Nuestro interlocutor podría, ante tal pregunta, responder algo como: "Pienso que no lo tienen porque la violencia no es un medio legítimo para obtener derechos." Dada la respuesta de nuestro interlocutor, que es un intento, tal vez no completamente exitoso, tal vez confuso, de formular el principio $P$, podríamos afirmar: "Es precisamente por esa razón que debemos rechazar que un gobernante que somete violentamente a otro tenga derecho a mandar sobre él”. Así, con nuestra pregunta, estaríamos induciendo a nuestro interlocutor a admitir que la razón más plausible para creer que $A$ es $w$ es la aceptación de $P$, pero también que $P$ implica que $B$ es $w$. Y llegados aquí, podríamos finalmente señalar que, si somos congruentes, debemos admitir que $B$ es $w$.

Tanto para Govier como para Waller, del principio universal que una analogía a priori presupone (o "implica") se sigue deductivamente su conclusión. A diferencia de Govier, sin embargo, Waller sostiene que el elemento análogo que figura en las premisas de estos argumentos - esto es, el hecho de que $A$ es $w$ - tiene la función de llamar nuestra atención sobre el principio en cuestión y de guiarnos hacia él, pero sobre todo de mostrarnos que dicho principio es, no uno que la persona que argumenta nos está pidiendo aceptar, sino uno que se encuentra ya entre los principios universales que aceptamos. Para Waller, nuestra aceptación del principio $P$ nos es revelada cuando admitimos que $A$ es $w$ y cuando captamos que la razón más plausible que tenemos para admitir que $A$ es $w$ es, precisamente, la aceptación de $P$. Así, de acuerdo con Waller, al aceptar que una analogía a priori es correcta no hacemos fundamentalmente otra cosa más que reconocer nuestros propios "valores y creencias profundos" (Waller, 2001, p. 208). Dado el funcionamiento de las analogías a priori y el papel central que en ellas juega el principio $P$, Waller sostiene que es incorrecto relegar este principio a la categoría de aserción meramente "implicada". Para Waller, $P$ es una parte integral de estos argumentos y debe ser incluido entre sus premisas. Una vez incluido el principio $P$ entre las premisas de las analogías a priori - tal como lo muestra el esquema de Waller - se puede apreciar que dichas analogías son esencialmente argumentos deductivos.

Desde luego, Waller no supone, como hemos visto, que sea fácil identificar y formular el principio universal implícito en una analogía a priori. Para Waller, el elemento análogo tiene la función de guiarnos en la identificación de dicho principio, pero no de manera directa, sino a través de un proceso de "ajustamiento mutuo" entre la formulación tentativa del principio y las particularidades de cada caso (Waller, 2001, p. 208). Por ejemplo, en la 
analogía a priori de Locke, vimos que la consideración del elemento análogo nos permite obtener una primera formulación del principio universal que el argumento presupone, a saber: la violencia no es un medio legítimo para obtener derechos. Esta formulación puede, sin embargo, ser objetada. Tal vez el principio universal identificado tiene excepciones relevantes, tal vez no es aplicable de la misma manera a ladrones y piratas, por un lado, y a gobernantes, por el otro. La consideración de las particularidades de cada caso, o de nuevos casos análogos, puede llevarnos a revisar nuestra formulación inicial. Sin embargo, para Waller, el hecho de que un tal proceso de ajustamiento mutuo sea necesario no debe hacernos creer que las analogías a priori no son argumentos deductivos. Tales procesos de ajustamiento mutuo son inevitables en la medida en que los principios universales que actúan sobre nuestra manera de concebir ciertos fenómenos y de razonar sobre ellos no son fácilmente accesibles. Esta situación no implica que los argumentos analógicos sean de un tipo distinto de los argumentos deductivos, sino únicamente que el uso de analogías en la argumentación es difícilmente eliminable, pues se trata de herramientas indispensables para acceder a los principios que implícitamente aceptamos y que influyen en nuestra manera de formular y evaluar argumentos.

En su respuesta a Waller, Govier (2002) sostiene que, precisamente, en la medida en que un proceso como el que describe Waller es necesario para acceder al principio $P, P$ no puede ser una premisa implícita. Una premisa implícita, de acuerdo con Govier, tendría que ser mucho más accesible y demandar mucho menos esfuerzo de creatividad para ser formulada (Govier, 2002). Govier admite que un proceso de ajustamiento mutuo es de un gran valor y permite entender la importancia de las analogías a priori en el desarrollo de pensamiento crítico, pero rechaza que eso sea una razón para aceptar una reconstrucción deductivista de estos argumentos. Para Govier, Waller no logra distinguir entre "un argumento per se, tal como es articulado por su autor, y una reconstrucción filosófica de este argumento" (Govier, 2002, p. 155), de ahí que asimile erróneamente las analogías a priori a argumentos deductivos.

Una vez presentadas las propuestas de Govier y Waller, expondré mi propia posición en el debate. Como vimos, ambos autores sostienen, aunque en sentidos aparentemente distintos, que las analogías a priori presuponen o "implican" principios de carácter universal. Mi propuesta parte igualmente de esta idea. Así, la pregunta que debemos plantearnos es la siguiente: ¿de qué forma son presupuestos los principios de carácter universal implícitos en las analogías a priori? En concordancia con Govier, sostengo que dichos principios no deben ser considerados premisas implícitas. Sin embargo, junto con Waller, sostengo que las analogías a priori son asimilables a argumentos deductivos. Como señalé en la sección anterior, sostengo que, además de la información de trasfondo y de las premisas implícitas, 
existe efectivamente una tercera forma de presuponer información en un argumento que no considero que sea la que Govier haya podido tener en mente. Se trata de conocimiento implícito que constituye las normas y restricciones que regulan la aplicación de conceptos. De acuerdo con mi propuesta, los principios de carácter universal implícitos en las analogías a priori son presupuestos de esta manera por las personas que argumentan. En la siguiente sección, explicaré más precisamente en qué consiste esta tercera forma de presuponer información en un argumento. Basándome en las ideas que ahí discutiré, sin embargo, intentaré mostrar, en la sección IV, que las analogías a priori no constituyen un tipo de argumento distinto de los argumentos deductivos.

\section{Aplicación de conceptos y argumentación}

En la elaboración de mi propuesta, parto del hecho de que poseemos una capacidad intuitiva de argumentar lógicamente. En otras palabras, sostengo que somos intuitivamente capaces de identificar y formular buenas razones para respaldar o refutar aserciones en un intercambio argumentativo. La cuestión es de dónde surge esta capacidad. La tesis que defiendo es que el factor principal (aunque ciertamente no el único) que explica nuestra capacidad intuitiva de argumentar lógicamente es nuestra sensibilidad a las condiciones de aplicación de nuestros conceptos. Dicha sensibilidad es básicamente aquello en lo que consiste ser capaz de formar juicios, pues ser capaz de formar juicios es ser capaz de aplicar conceptos (los conceptos que constituyen el contenido de los juicios) y ser capaz de aplicar un concepto es ser sensible a sus condiciones de aplicación, es decir ser sensible a las normas y restricciones que determinan cuándo el concepto puede o debe ser aplicado y cuándo no puede o no debe ser aplicado (Bernache Maldonado, 2018, 2019). Para ilustrar estas ideas, consideremos uno de los esquemas de argumentación propuestos por Douglas Walton (2013; véase también Walton et al. 2008):

Premisa mayor: el informante $E$ es un experto en el campo de conocimiento $C$ el cual contiene la proposición $p$.

Premisa menor: $E$ afirma que $p$ es verdadera (falsa).

Conclusión: $p$ es verdadera (falsa).

Este esquema - el esquema por opinión de experto - representa un tipo de argumento que podemos considerar racional. ¿Pero cómo explicar tal racionalidad? La clave está en comprender que toda persona que juzga que un individuo $E$ es un experto se compromete forzosamente con la verdad de las siguientes dos afirmaciones: (a) que $E$ es una autoridad 
en un campo específico de conocimiento y (b) que el tipo de autoridad que $E$ posee en su campo de conocimiento constituye una buena razón para confiar en su capacidad de juzgar acerca de la verdad o falsedad de una proposición que pertenece a dicho campo. Ahora bien, imaginemos una persona que argumenta empleando el esquema por opinión de experto. ¿De qué forma se compromete la persona con la verdad de (a) y (b)? ¿Presupone (a) y (b) como premisas implícitas? ¿O bien como información de trasfondo?

La idea que defiendo es que la persona no presupone (a) y (b) ni como premisas implícitas ni como información de trasfondo, sino que la aceptación tácita de (a) y (b) manifiesta la influencia en su razonamiento del conocimiento implícito de las condiciones de aplicación del concepto experto, es decir de las normas y restricciones que determinan cuándo este concepto puede o debe ser aplicado y cuándo no puede o no debe ser aplicado. En la medida en que admitimos que la persona es capaz de aplicar el concepto experto, es decir de juzgar que un determinado individuo es un experto, debemos igualmente asumir que la persona es sensible a las condiciones de aplicación de este concepto. Por ejemplo, si constatamos que la persona parece plenamente juzgar que $E$ es un experto en el campo de conocimiento $C$, pero que al mismo tiempo rechaza la autoridad de $E$ en tanto que experto en dicho campo, podemos naturalmente suponer que la persona sabe algo acerca de $E$, o acerca de su situación en ese momento específico, que permite dudar de la sinceridad o de la libertad con la que $E$ se expresa, o que desacredita de algún otro modo su testimonio. Sin embargo, si la persona no posee información alguna de este tipo, sino que simplemente es incapaz de ver la conexión que existe entre el hecho de admitir que $E$ es un experto en el campo $C$ y la aceptación de la autoridad de $E$ en ese campo, la conclusión más razonable que podemos extraer es que la persona no sabe realmente en qué consiste ser un experto o, dicho de otro modo, que no posee el concepto experto. En semejante situación, lo que constatamos es que la persona no es sensible a las condiciones de aplicación de este concepto, pues no manifiesta las disposiciones racionales que esperaríamos de alguien que lo posee auténticamente y que es, por consiguiente, verdaderamente capaz de juzgar que un determinado individuo es un experto.

Aunque es difícil formular criterios precisos que en cada caso nos permitan distinguir entre la información presupuesta en un argumento como información de trasfondo y aquella que es presupuesta como premisas implícitas, para a su vez poder distinguir entre estos dos tipos de información tácita y la información presupuesta como condiciones de aplicación de un concepto, ciertas características generales pueden ser señaladas. Consideremos, por ejemplo, el siguiente argumento: 
Premisa: $E$ es un experto en el campo de conocimiento $C$.

Conclusión: $E$ tiene títulos universitarios.

Una persona que emplea un tal argumento admite tácitamente que los expertos tienen títulos universitarios (o, al menos, aquellos que lo son en el campo $C$ ), pues de otra forma no podría derivar la conclusión. Esta información presupuesta constituye una premisa implícita. ¿Pero qué permite afirmarlo? En general, asumimos que las premisas de un argumento son lógicamente independientes unas de otras: si $p$ y $q$ son las premisas de un argumento, suponemos entonces que $p$ no implica $q$ o la negación de $q$ y que $q$ no implica $p$ o la negación de $p$. De lo contrario, $p$ y $q$ constituirían un conjunto redundante o inconsistente de premisas. Así, dado que las aserciones " $E$ es un experto en el campo de conocimiento $C$ " y "los expertos tienen títulos universitarios" son lógicamente independientes una de otra (y que la segunda es tácitamente asumida en el argumento), podemos afirmar que ambas constituyen premisas de nuestro pequeño argumento: la primera está explícitamente formulada, mientras que la segunda es implícita.

En cambio, la aserción " $E$ es un experto en el campo de conocimiento $C$ ” no es lógicamente independiente ni de (a) ni de (b): una vez que hemos admitido $E$ es un experto en $C$, estamos obligados a reconocer el tipo específico de autoridad que $E$ posee en su campo de conocimiento o, si no estamos dispuestos a reconocer tal autoridad (sin tener información adicional que la ponga en duda), debemos rechazar que $E$ sea un experto en $C$. De ahí que no sea plausible admitir que (a) y (b) constituyen premisas implícitas del esquema por opinión de experto.

¿Pero no podrían expresar (a) y (b) mera información de trasfondo? La información de trasfondo presupuesta en un argumento no puede ser información lógicamente independiente de sus premisas, pues de otra manera prácticamente cualquier información irrelevante que no es enunciada podría ser considerada información de trasfondo presupuesta en el argumento. En general, la información de trasfondo es aquella con la cual la persona que argumenta se compromete ineludiblemente cuando afirma las premisas de su argumento, o cuando asume que es posible derivar la conclusión de dichas premisas, pero que no es considerada información particularmente polémica. Por ejemplo, una persona que emplea el esquema por opinión de experto, al sostener su premisa menor, es decir al sostener que $E$ afirma que $p$ es verdadera (falsa), se compromete ineludiblemente con la proposición según la cual los expertos son personas capaces de comunicar su testimonio. En la medida en que su interlocutor no pone en duda el hecho de que los expertos sean personas capaces de comunicar su testimonio, sino que lo admite sin dificultad, tal información implícitamente asumida puede ser considerada mera información de trasfondo. Desde luego, la informa- 
ción de trasfondo puede ser cuestionada con el fin de refutar el argumento que la presupone. En tal caso, dicha información deja de ser mera información de trasfondo y se integra plenamente al proceso dialéctico de la argumentación.

De acuerdo con los criterios mencionados, (a) y (b) pueden ser consideradas aserciones que expresan información de trasfondo presupuesta en el esquema por opinión de experto, pues ninguna de ellas es lógicamente independiente de las premisas del esquema (en particular de la premisa mayor) y ninguna de ellas es particularmente polémica. Tal resultado no es sorprendente, dada la laxitud de los criterios con los que clasificamos información implícita en un argumento, o en una simple afirmación, como información de trasfondo. Sin embargo, hay una diferencia importante entre la mera información de trasfondo y la información que constituye conocimiento implícito de las condiciones de aplicación de un concepto. La información que es presupuesta en un argumento como mera información de trasfondo puede ser de carácter muy general y su contenido puede ser muy variado. Un ejemplo es el principio de no contradicción que, como Govier señala, es presupuesto como información de trasfondo en todo argumento deductivo, aunque también en aquellos argumentos que resultan del esquema por opinión de experto. Otro ejemplo de información de trasfondo que este esquema presupone es el de la proposición según la cual el mensaje que produce un emisor no se transforma aleatoriamente en un mensaje distinto en el lapso de tiempo que tarda en llegar al receptor. A diferencia de la mera información de trasfondo, la información presupuesta como conocimiento implícito de las condiciones de aplicación de un concepto tiene límites mucho más restringidos.

Como fue mencionado, ser capaz de formar un juicio es ser capaz de aplicar los conceptos que constituyen su contenido; y esta última capacidad implica a su vez la sensibilidad a las condiciones particulares de aplicación de estos conceptos. Poseer este tipo peculiar de sensibilidad es básicamente aquello en lo que consiste ser capaz de aplicar conceptos. Las disposiciones racionales que una persona manifiesta dado el hecho de que es sensible a las condiciones de aplicación de un determinado concepto pueden ser muy variadas. Sin embargo, en muchos casos, no es difícil identificar un núcleo de disposiciones racionales básicas que, en determinadas circunstancias, toda persona que posee auténticamente el concepto tendría que manifestar. Tal es el caso de la aceptación de la autoridad de $E$ en su campo de conocimiento, una vez que hemos juzgado que $E$ es un experto y en la medida en que no poseemos información adicional que, de algún modo, ponga en entredicho esta autoridad. En estas condiciones, negar la autoridad de $E$ equivaldría a asumir sin más que los expertos, en tanto que expertos, no poseen autoridad alguna en sus propios campos de conocimiento. De alguien que asume tal cosa podemos afirmar que no posee realmente el concepto experto, es decir que no es auténticamente capaz de juzgar que un determinado 
individuo es un experto. En cambio, al negar mera información de trasfondo - como, por ejemplo, el hecho de que los expertos sean personas capaces de comunicar su testimonio - una persona puede asumir una idea muy difícil de defender, y en este sentido absurda, pero no hace en general algo que ponga en duda sus competencias conceptuales. En pocas palabras, la información presupuesta como conocimiento implícito de las condiciones de aplicación de un concepto, a diferencia de la mera información de trasfondo, es conocimiento constitutivo de la posesión de capacidades conceptuales.

Una capacidad, de cualquier tipo que sea, presupone siempre conocimiento constitutivo de su propia posesión. Por ejemplo, una persona capaz de andar en bicicleta, de dirigir un dron o de tocar un instrumento musical dispone forzosamente de conocimiento constitutivo de la posesión de estas capacidades, es decir conocimiento generalmente gradual que es adquirido con la adquisición de la capacidad misma y que constituye tal adquisición. Desde luego, el conocimiento constitutivo de la posesión de una capacidad no es necesariamente formulable de manera explícita por la persona misma que lo posee y no tiene necesariamente una forma proposicional, sino que se manifiesta fundamentalmente en la manera intuitiva y competente en que la persona regula el ejercicio de su propia capacidad en cada ocasión concreta. En el caso específico de la capacidad de aplicar conceptos, tal forma de regulación intuitiva y competente no es otra cosa que la sensibilidad a las condiciones particulares de aplicación de conceptos.

De acuerdo con la propuesta que defiendo, las condiciones de aplicación del concepto experto explican la racionalidad del esquema por opinión de experto y la posesión de la capacidad de aplicar este concepto explica el hecho de que seamos capaces de captar tal racionalidad. En efecto, en la medida en que (a) y (b) reflejan la posesión de conocimiento constitutivo de la capacidad de aplicar el concepto experto, ambas aserciones revelan, no mera información de trasfondo, sino información presupuesta como conocimiento implícito de las condiciones de aplicación de este concepto. Así, dado que ser capaz de aplicar un concepto es ser sensible a sus condiciones de aplicación, una persona capaz de aplicar el concepto experto, en virtud de la posesión de esta capacidad, debe igualmente ser capaz de captar la racionalidad del esquema por opinión de experto y de evaluar sus distintas aplicaciones. Pero el esquema por opinión de experto es solamente un ejemplo. De manera general, como ya fue mencionado, sostengo que la sensibilidad a las condiciones de aplicación de nuestros conceptos es el factor principal que explica nuestra capacidad intuitiva de argumentar lógicamente, es decir nuestra capacidad intuitiva de identificar y formular buenas razones para respaldar o refutar aserciones en un intercambio argumentativo.

El hecho de afirmar que los conceptos tienen condiciones de aplicación no nos obliga a asumir que existen definiciones de nuestros conceptos. La información expresada por las 
aserciones (a) y (b) no debe necesariamente ser vista como una definición del concepto experto. No obstante, en la medida en que admitimos que, para cada concepto, existe conocimiento constitutivo de la posesión de la capacidad de aplicarlo, debemos igualmente admitir que, para cada concepto, existe un núcleo de disposiciones racionales básicas que manifiestan dicho conocimiento y, con ello, la posesión de dicha capacidad.

Las disposiciones racionales básicas asociadas a la capacidad de aplicar un concepto pueden ser vistas como elementos que permiten, no solamente determinar cuándo una persona es capaz de aplicar el concepto, sino también individuar el concepto mismo, es decir especificar aquello que hace que el concepto en cuestión sea distinto de todos los demás. Tal es la propuesta de Christopher Peacocke en su libro seminal A study of concepts (Peacocke, 1992). Sin embargo, nada de lo dicho aquí nos obliga a aceptar esta propuesta. Es posible sostener que el conocimiento constitutivo de la capacidad de aplicar un concepto es información asociada al concepto que condiciona, en todo contexto, sus distintas aplicaciones, sin que sea necesario asumir que dicha información permite siempre su individuación. Incluso, no es necesario asumir que, para cualquier información asociada a un concepto, siempre es posible determinar si se trata o no de conocimiento constitutivo de la capacidad de aplicarlo. En el caso del concepto experto, admitimos que las aserciones (a) y (b) reflejan conocimiento constitutivo de la capacidad de aplicarlo, mientras que la aserción "los expertos tienen títulos universitarios" no refleja conocimiento de este tipo. Sin embargo, nada nos impide reconocer la existencia de información asociada a un concepto a la que es difícil, o incluso imposible, aplicar esta distinción.

Una propuesta de este tipo es defendida por Edouard Machery $(2009,2015)$ quien sostiene que un concepto es un núcleo estable de información almacenado en la memoria de largo plazo que es empleado en operaciones cognitivas de orden superior (categorización, juicio, razonamiento). De acuerdo con Machery, la información que constituye el núcleo estable de un concepto es información asociada al concepto que, cuando este es requerido, es activada y accesible por defecto, esto es: de forma rápida, automática e independiente del contexto (Machery, 2015). Aunque la automaticidad ha sido generalmente considerada una propiedad discreta de un proceso cognitivo, Machery señala que puede también ser vista como una magnitud continua, tal como la rapidez, y lo mismo sostiene respecto de la independencia del contexto. En este caso, nos dice Machery, la información perteneciente al núcleo estable de un concepto puede ser caracterizada como aquella cuya activación es efectuada, cuando el concepto es requerido, según los valores de cada una de estas magnitudes situados, respectivamente, en los extremos rápido, automático e independiente del contexto (Machery, 2015). Esta caracterización del núcleo estable de un concepto deja abierta la posibilidad de casos en los que es difícil, o incluso imposible, determinar si el 
valor de la activación de cierta información asociada a un concepto, en cualquiera de sus magnitudes, es suficientemente extremo como para admitir que dicha información pertenece a su núcleo estable.

Es importante resaltar que los criterios que emplea Machery para determinar cuándo la información asociada a un concepto pertenece a su núcleo estable son criterios puramente estadísticos: toda información asociada a un concepto que sea accesible, cuando el concepto es requerido, de forma rápida, automática e independiente del contexto será información perteneciente a su núcleo estable. Criterios de este tipo son empleados en las teorías psicológicas en las que Machery se basa para construir su propuesta, como la teoría de prototipos (Smith \& Medin, 1981) o la teoría de ejemplares (Brooks, 1978; Nosofsky, 1986). Una consecuencia del uso de tales criterios es que, con ellos, es claro de qué forma un concepto puede ser intrapersonalmente individuado, pero no es obvio que la individuación interpersonal de conceptos sea posible. Para Machery, sin embargo, tal situación no constituye un problema, pues individuar interpersonalmente nuestros conceptos no sería, según él, necesario para la psicología (Machery, 2009).

No obstante, cuando argumentamos, no formulamos razones para nuestro consumo propio, sino que las sometemos a la evaluación de los demás y evaluamos las razones que los demás formulan (Mercier \& Sperber, 2017). Un argumento que una persona formula basándose en información que asocia firmemente a un concepto puede ser rechazado por otra persona que niega (de forma implícita o explícita) la firmeza de esta asociación. Si aplicáramos los criterios estadísticos de Machery y descubriéramos que, en el caso de la persona que formula el argumento, la información pertenece al núcleo estable del concepto, mientras que, en el caso de la otra persona, esto no es así, ¿qué podríamos decir al respecto? Si aceptáramos las ideas de Machery, tendríamos que decir que las personas no están empleando el mismo concepto y que no existe, por consiguiente, desacuerdo real entre ellas. Sin embargo, aunque en algunos casos esto puede ser cierto, admitimos también la posibilidad de que una de las dos personas se equivoque y la otra esté en lo correcto. En otras palabras, admitimos que el uso de conceptos está sometido a normas y restricciones que se aplican interpersonalmente.

Muchos de los datos en los que se basan las distintas teorías de conceptos en psicología cognitiva son extraídos de experimentos sobre nuestra actividad de categorización (Machery, 2009; Prinz, 2002; Smith \& Medin, 1981). En estos experimentos, las personas reaccionan clasificando un estímulo cuyas propiedades han sido cuidadosamente controladas. Tales reacciones aportan información acerca de la manera en que aplicamos conceptos y su influencia está presente, sin duda, en nuestra actividad argumentativa. Sin embargo, cuando argumentamos, nuestras reacciones no son simplemente constatadas y aceptadas, sino que son ante todo sometidas a evaluación. La información que una persona asocia firmemente a 
un concepto puede explicar algunas de sus reacciones cuando aplica el concepto, entre ellas la formulación de un determinado argumento. Al formular su argumento, sin embargo, la persona somete su uso del concepto a una evaluación interpersonal: otra persona puede rechazar el argumento y mostrar que es un error asociar la información en cuestión a dicho concepto. De esta forma pueden establecerse interpersonalmente las normas y restricciones que regulan la aplicación de conceptos. Así, el fenómeno de la argumentación muestra que los conceptos no pueden ser estudiados y caracterizados de forma meramente estadística e intrapersonal, sino que nuestra actividad conceptual es fundamentalmente una actividad normativa e interpersonal (Brandom, 1994).

\section{Analogías a priori}

¿De qué forma estos planteamientos pueden ayudarnos a comprender la naturaleza de las analogías a priori? Como hemos visto, todo argumento conlleva conocimiento implícito de las condiciones de aplicación de conceptos. Cuando empleamos un argumento, de cualquier tipo que sea, nuestro propósito es inducir en nuestro interlocutor la formación de un determinado juicio o la revisión de un juicio previamente formado, o bien resistir a la revisión de nuestros propios juicios. Nuestra sensibilidad a las condiciones de aplicación de nuestros conceptos nos permite identificar las razones que, en un determinado contexto, respaldan o impiden su aplicación y, por consiguiente, la formación de los juicios en cuyo contenido figuran. Las analogías a priori no son, respecto de estas características, argumentos distintos de los demás. Una persona que formula una analogía a priori intenta mostrar que, en el contexto relevante para su argumentación, los conceptos que constituyen el contenido de la conclusión deben ser aplicados.

Una vez más, veamos la analogía a priori de Locke. Según lo que plantea Locke, es moralmente ilegítimo que un gobernante mande sobre otro a quien ha sometido por la fuerza. El concepto principal cuya aplicación pretende Locke inducir en sus lectores es el concepto moralmente ilegítimo, pues el objetivo principal de su argumentación es que juzguemos, como él mismo lo hace, que la situación que él nos describe es, en efecto, moralmente ilegítima. De acuerdo con mi propuesta, para lograr su propósito, Locke debe basar sus razones en las condiciones de aplicación del concepto moralmente ilegítimo. En la medida en que es sensible a las condiciones de aplicación de este concepto, Locke debe poder identificar las razones que busca y nosotros, en la medida en que también poseemos esta forma de sensibilidad, debemos poder evaluarlas. En esto, el funcionamiento de una analogía a priori no es distinto del funcionamiento de cualquier otro argumento (particularmente de los argumentos deductivos, como veremos). 
Ahora bien, ¿cuáles son las condiciones de aplicación del concepto moralmente ilegíti$m o$ ? En otras palabras, ¿cuáles son las normas y restricciones que regulan la aplicación de este concepto y que son constitutivas de la capacidad misma de aplicarlo? Para varios autores, los conceptos morales permiten esencialmente a quienes los emplean expresar sus emociones o actitudes de rechazo o aprobación ante diversas acciones o situaciones (Ayer, 1952; Blackburn, 1998; Gibbard, 1990). Otros autores, en cambio, defienden una u otra forma de realismo moral (Peacocke, 2004). Ninguna de estas posturas es incompatible con la existencia de normas y restricciones que regulan la aplicación de conceptos morales, ni con el hecho de que algunas de estas normas y restricciones sean constitutivas de la capacidad de aplicarlos, al menos tal como son utilizados en una determinada comunidad. Aun así, es preciso reconocer que aquello que es considerado moralmente ilegítimo no es siempre lo mismo de una época a otra, o incluso de una generación a la siguiente, ni tampoco entre personas de distintos grupos socioeconómicos. Sin duda, los juicios morales se encuentran entre aquellos que generan mayor desacuerdo entre los miembros de una misma comunidad. Mi postura es que es precisamente en este tipo de casos que el uso de analogías a priori es particularmente útil.

Si queremos formular una analogía a priori con el propósito de inducir en nuestro interlocutor la aplicación del concepto moralmente ilegítimo a una situación $S$, debemos comenzar describiendo una situación $S A$ en la que es claro, al menos para nosotros y para nuestro interlocutor, que el concepto debe ser aplicado. Como señala Govier, tal situación no tiene forzosamente que ser real, sino que debe ser ante todo una situación que de manera firme y consensual consideremos moralmente ilegítima. Desde luego, además de ser considerada moralmente ilegítima, $S A$ debe ser análoga a $S$. La similitud entre $S$ y $S A$ debe ser relevante para la aplicación del concepto moralmente ilegítimo, esto es: las características de SA que activan nuestras disposiciones racionales y nos conducen a juzgar que $S A$ es una situación moralmente ilegítima deben estar presentes en $S$. Estas características no tienen necesariamente que ser claras para nosotros, como hemos visto, pues basta con que podamos sentirnos persuadidos de que el concepto moralmente ilegítimo debe ser aplicado, por las mismas razones, tanto a $S A$ como a $S$, aun sin saber exactamente cuáles son esas razones. Una vez identificada una tal situación $S A$, podemos presentarla como una razón para juzgar que $S$ es una situación moralmente ilegítima. Nuestra sensibilidad a las condiciones de aplicación del concepto moralmente ilegítimo puede guiarnos, de este modo, en la formulación de nuestra analogía a priori, aun si no es claro para nosotros cuáles son las normas y restricciones que determinan nuestras disposiciones racionales.

En su argumentación, Locke ilustra este procedimiento, pues nos presenta el caso de los ladrones y piratas como un ejemplo evidente de una situación moralmente ilegítima, 
pero también como un caso que, dadas sus características, debería hacernos juzgar que es moralmente ilegítimo, por las mismas razones, que un gobernante mande sobre otro a quien ha sometido por la fuerza.

Nuestra sensibilidad a las condiciones de aplicación del concepto moralmente ilegítimo debe también permitirnos evaluar la analogía a priori de Locke. Observemos, en primer lugar, que es claro que todo acto de ladrón o de pirata, en tanto que acto de ladrón o de pirata, es moralmente ilegítimo. También es claro que no todo acto de gobernante, en tanto que acto de gobernante, es moralmente ilegítimo. Nuestra sensibilidad a las condiciones de aplicación de los conceptos moralmente ilegítimo, ladrón, pirata y gobernante nos permite captar la verdad de estas afirmaciones. Ahora bien, notemos que el hecho de someter a alguien por la fuerza y obligarlo a obedecernos puede ser moralmente ilegítimo, pero no necesariamente lo es, pues no es difícil imaginar circunstancias en las que sería incluso moralmente incorrecto no someter a una persona de esta manera. Así, el sometimiento que ladrones y piratas, en tanto que ladrones y piratas, ejercen sobre otros no es comparable con el sometimiento que un gobernante, en tanto que gobernante, puede ejercer sobre otro gobernante, aun si se trata de un sometimiento por la fuerza, pues este último, a diferencia del primero, podría ser moralmente correcto. La analogía a priori de Locke no parece establecer su conclusión y nuestra sensibilidad a las condiciones de aplicación del concepto moralmente ilegítimo nos permite verlo.

Cuando la aplicación de un concepto tiene consecuencias sociales importantes, podemos esperar el surgimiento de fuertes polémicas acerca de si es correcto o no aplicarlo en una determinada situación. Los conceptos morales suelen causar tales polémicas, pero otros conceptos evaluativos pueden también generarlas, como el concepto estrategia correcta aplicado a acciones que se pretende implementar en, por ejemplo, una crisis económica. En circunstancias semejantes, argumentar empleando analogías puede ser muy útil, pues es un procedimiento que permite describir un caso consensual que, respecto de las características que producen la aplicación del concepto, es comparable con el caso polémico. Esta comparación activa nuestra sensibilidad a las condiciones de aplicación del concepto y puede influir en nuestras disposiciones racionales de manera que el caso polémico sea juzgado como el caso consensual.

Cuando Waller afirma que las analogías a priori tienen la función de llamar nuestra atención sobre principios que revelan nuestros "valores y creencias profundos", parece enforcarse exclusivamente en el uso de estos argumentos para inducir la aplicación de conceptos morales. Pero, como fue mencionado, las analogías a priori pueden ser empleadas para inducir la aplicación de conceptos de otro tipo. Igualmente, aunque las analogías a priori puedan tener, en ciertos casos, el efecto de llamar nuestra atención sobre principios (morales u otros) que 
implícitamente aceptamos, no hay razón para suponer que tal sea su función. El propósito de una persona que formula una analogía a priori, o cualquier otro tipo de argumento, es inducir la aplicación de un determinado concepto. Para ello, la persona debe basar sus razones en las condiciones de aplicación del concepto en cuestión. Si el concepto es moral, sus condiciones de aplicación tendrán que ver con principios morales. Sin embargo, si no es moral, no hay razón para suponer que sus condiciones de aplicación tendrán algo que ver con tales principios.

Los principios que influyen en la formulación y evaluación de analogías a priori, así como en la formulación y evaluación de cualquier otro tipo de argumento, son esencialmente las normas y restricciones que regulan la aplicación de nuestros conceptos. El esquema con el que Waller pretende representar la estructura de las analogías a priori contempla la inclusión, entre las premisas de estos argumentos, de principios que, según mi planteamiento, constituyen condiciones de aplicación de conceptos. Sin embargo, aunque estos principios pueden ciertamente ser explicitados y utilizados como premisas en un argumento, no es la función de una analogía a priori incitar a su explicitación. Sin contar que el argumento resultante seguiría implicando, como todo argumento, información presupuesta como conocimiento implícito de las condiciones de aplicación de conceptos, la cual no estaría integrada entre sus premisas. En general, la función de un argumento, del tipo que sea, no es llamar nuestra atención sobre las normas y restricciones que regulan la aplicación de nuestros conceptos, sino basarse en ellas para establecer su conclusión, es decir para mostrar que es correcto, en una determinada situación, aplicar los conceptos que constituyen su contenido. De ahí que el análisis de Waller deba ser rechazado.

No obstante, esto no implica que las analogías a priori constituyan un tipo distinto de los argumentos deductivos. Como en el caso de cualquier otro argumento, una persona que formula una analogía a priori intenta inducir en su interlocutor la aplicación de un determinado concepto (y, por consiguiente, la formación de un determinado juicio) basándose esencialmente (aunque no exclusivamente) en las condiciones particulares de aplicación de dicho concepto. Esto es cierto incluso de los argumentos más puramente deductivos. Por ejemplo, la conclusión $\sim(p \& q)$ se sigue de la premisa $\sim p$ en virtud de las normas y restricciones que regulan la aplicación de los conceptos conjunción y negación. A esto se podría tal vez objetar que las analogías a priori, a diferencia de los argumentos puramente deductivos, no establecen su conclusión de forma definitiva, sino que esta es siempre retractable (Bermejo-Luque, 2012; Freeman, 2013; Juthe, 2020). Pero la posibilidad de establecer de forma definitiva la conclusión de un argumento depende ante todo de las condiciones de aplicación de los conceptos que constituyen el contenido de premisas y conclusión, más que de las estrategias que empleamos para inducir la aplicación de conceptos. Así, no hay razón para suponer que una analogía $a$ priori no pueda establecer de forma definitiva su conclusión. 
Un ejemplo puede ser el siguiente. Imaginemos que intentamos defender la proposición según la cual no se puede ser un experto si no se tiene cierta autoridad en un campo específico de conocimiento. El concepto principal cuya aplicación deseamos inducir en nuestro interlocutor es la negación del concepto modal posible (aplicado a la situación de ser un experto sin tener la autoridad mencionada). Para lograr nuestro propósito, podemos hacer una analogía entre ser un experto y ser un ciclista profesional. Podemos señalar, por ejemplo, que así como es absurdo admitir que alguien sea un ciclista profesional si negamos que alguna vez haya aprendido a andar en bicicleta, es absurdo admitir que alguien sea un experto si rechazamos que tenga autoridad en un campo específico de conocimiento. Tal comparación puede activar la sensibilidad de nuestro interlocutor a las condiciones de aplicación del concepto modal posible y hacerle ver que, por la misma razón que niega que puede haber ciclistas profesionales que nunca aprendieron a andar en bicicleta, debe negar que puede haber expertos sin autoridad en un campo de conocimiento. La conclusión de nuestra analogía a priori es establecida de forma definitiva, pero esto tiene fundamentalmente que ver con las condiciones de aplicación del concepto experto, no con nuestra estrategia para inducir en nuestro interlocutor la aceptación de dicha conclusión.

El punto que deseo resaltar es el siguiente: no existe un tipo de inferencia o de respaldo racional que podamos considerar específicamente analógico. Mi conclusión no es que las analogías a priori sean "meros" argumentos deductivos, sino que los principios de fundamentación racional que operan en las analogías a priori son esencialmente los mismos que los que operan en la argumentación deductiva. La manera específica en que las condiciones de aplicación de conceptos hacen funcionar los argumentos deductivos es, como vimos, exactamente la misma que en el caso de las analogías a priori. No hay razón para suponer, por consiguiente, que las analogías a priori revelen una forma sui géneris de fundamentación de ideas. En esto, mi desacuerdo es claro con autores como André Juthe. Según Juthe, la particularidad de los argumentos analógicos es que su funcionamiento no requiere compromiso alguno con premisas universales (Juthe, 2005, 2015, 2020). Sin embargo, como fue señalado, todo argumento - incluyendo las analogías a priori - conlleva el uso de conocimiento implícito de las condiciones de aplicación de conceptos, es decir conlleva el uso de principios universales o generales de cierto tipo. Juthe señala categóricamente que "la premisa universal necesaria para transformar una argumentación analógica en un argumento deductivo no puede ser aportada" (Juthe, 2020: 278) y añade que, en los casos en los que la premisa en cuestión es suministrada, la reconstrucción resulta en un argumento mucho peor que la versión analógica original.

No obstante, uno de los elementos centrales del análisis de Juthe es lo que él llama "la relación de determinación" (Juthe, 2020). Tal relación existe entre, por un lado, ciertas pro- 
piedades del elemento análogo empleado en un argumento analógico y, por el otro, cierto predicado $P$. En virtud de esta relación, según Juthe, las propiedades en cuestión determinan la asignación del predicado $P$ al elemento análogo. Cuando se establece, en un argumento analógico, una relación de correspondencia uno a uno entre las propiedades mencionadas del elemento análogo y ciertas propiedades del elemento meta, la relación de determinación se transfiere del elemento análogo al elemento meta y la asignación de $P$ a este último es posible. Es de esta forma que la conclusión del argumento puede ser derivada. El problema, sin embargo, es que la relación misma de determinación supone el uso de principios universales o generales, no como premisas implícitas, sino como normas y restricciones que regulan la aplicación de conceptos, pues la asignación de un predicado no es otra cosa que la aplicación de un concepto y la aplicación de un concepto implica, como ya fue mostrado, el conocimiento implícito de estas normas y restricciones. El hecho de que las condiciones generales de aplicación de nuestros conceptos no puedan, en muchos casos, ser explicitadas sin enfrentarse a dificultades importantes no implica que no jueguen un papel absolutamente esencial en el funcionamiento de las analogías $a$ priori, así como en el funcionamiento del resto de nuestros argumentos, particularmente de los argumentos deductivos.

Otros autores que, a diferencia de Juthe, reconocen la necesidad de principios universales en el funcionamiento de las analogías a priori intentan demostrar la particularidad de estos argumentos atribuyendo características especiales a dichos principios. Tal es el caso de Freeman (2013) quien sostiene que los principios que operan en las analogías a priori, a diferencia de los que operan en argumentos de otro tipo, son principios sintéticos a priori. Por mi parte, no veo ninguna razón para admitir que los principios que constituyen condiciones de aplicación de conceptos deban ser vistos como principios sintéticos a priori. De hecho, tampoco es necesario verlos como principios a priori. Las normas y restricciones que regulan la aplicación de conceptos son conocimiento constitutivo de la posesión de la capacidad de aplicarlos. No hay razón para suponer que tal conocimiento deba ser a priori o sintético a priori. Para afirmar tal cosa, es necesario discutir más profundamente el asunto. Pero, aun si admitiéramos que el conocimiento constitutivo de la capacidad de aplicar conceptos es conocimiento sintético a priori, no por ello habríamos identificado una propiedad distintiva de las analogías a priori, pues tal conocimiento actúa, como fue mencionado, en la formulación y evaluación de todo tipo de argumentos.

Así, aunque la formulación de analogías a priori sea una estrategia extremadamente útil para inducir la aplicación de conceptos en ciertas circunstancias especiales, no podemos por ello concluir que dichas analogías constituyen un tipo de argumento distinto de los argumentos deductivos. 


\section{Conclusión}

Partiendo de una tesis general acerca de nuestra capacidad intuitiva de argumentar lógicamente, he intentado mostrar que las analogías a priori no constituyen un tipo de argumento distinto de los argumentos deductivos. Las analogías a priori son argumentos particularmente eficaces en ciertas situaciones complejas, pero su funcionamiento no nos revela una forma sui géneris de fundamentación de ideas.

¿Pero qué decir de los otros tipos de argumentos analógicos? Aunque esta no ha sido la cuestión abordada en este artículo, podemos decir dos cosas al respecto. La primera es que, de acuerdo con Freeman (2013), es plausible reducir las analogías inductivas a argumentos inductivos convencionales. En cuanto al uso de analogías en argumentos abductivos, Marraud (2007) muestra que, en este tipo de casos, el objetivo es justificar cierta explicación de un fenómeno mostrando la semejanza de este fenómeno con otro para el cual una explicación similar es generalmente aceptada. Un tal tipo de argumento respalda su conclusión en virtud del principio abductivo según el cual "los casos similares deben recibir explicaciones similares" (Marraud, 2007, p. 177), y no en virtud de alguna forma propiamente analógica de respaldo racional. Desde una perspectiva retórica y dialéctica, la argumentación analógica se distingue como una estrategia singular e importante. Sin embargo, desde una perspectiva lógica, no parece razonable admitir que los argumentos analógicos sean argumentos de un tipo distinto por derecho propio.

\section{Referencias}

Alvargonzález, David. "Proposal of a classification of analogies." Informal Logic. 40(1) (2020): 109-137.

Ayer, Alfred. Language, truth, and logic. New York: Dover Publications, 1952.

Bermejo-Luque, Lilian. "A unitary schema for arguments by analogy." Informal Logic. 32(1) (2012): 1-24.

Bermejo-Luque, Lilian. "Deduction without dogmas: the case of moral analogical arguments." Informal Logic. 34(3) (2014): 311-336.

Bernache Maldonado, Fabián. "Condiciones de posesión de conceptos, racionalidad y argumentación." Revista Iberoamericana de Argumentación. 16 (2018): 1-32.

Bernache Maldonado, Fabián. “Conceptos y esquemas en la argumentación." Sincronía. 23(76) (2019): 105-135. 
Blackburn, Simon. Ruling passions. Oxford: Oxford University Press, 1998.

Brandom, Robert. Making it explicit. Cambridge, Massachusetts: Harvard University Press, 1994.

Brooks, Lee R. "Nonanalytic concept formation and memory for instances." En Rosch, E. y Lloyd, B. (eds.). Cognition and categorization (pp. 3-170). Hillsdale, N.J.: Lawrence Erlbaum Associates, 1978.

Freeman, James. "Govier's distinguishing a priori from inductive arguments by analogy: implications for a general theory of ground adequacy." Informal Logic. 33(2) (2013): 175-194.

Gibbard, Allan. Wise choices, apt feelings. Cambridge, Massachusetts: Harvard University Press, 1990.

Govier, Trudy. “Analogies and missing premises." Informal logic. 9(3) (1989): 141-152.

Govier, Trudy. "Should a priori analogies be regarded as deductive arguments?" Informal logic. 22(2) (2002): 155-157.

Govier, Trudy. A practical study of argument. Boston: Wadsworth, 2014.

Guarini, Marcello. "A defense of non-deductive reconstructions of analogical arguments." Informal Logic. 24(2) (2004): 153-168.

Juthe, André. "Argument by analogy." Argumentation. 19 (2005): 1-27.

Juthe, André. “Analogical argument schemes and complex argumentation." Informal logic. 35(3) (2015): 378-445.

Juthe, André. "A defense of analogy inference as sui generis." Logic and logical philosophy. 29(2) (2020): 259-309.

Locke, John. Segundo tratado sobre el gobierno civil. (Trad. de Carlos Mellizo) Madrid: Tecnos, 1689/2006.

Machery, Edouard. Doing without concepts. Oxford: Oxford University Press, 2009.

Machery, Edouard. "By default: concepts are accessed in a context-independent manner." En Margolis, E. y Laurence, S. (eds.). The conceptual mind (pp. 567-588). Cambridge, Massachusetts: M.I.T. Press, 2015. 
Marraud, Hubert. "La analogía como transferencia argumentative." Theoria. 59 (2007): 167-188.

Mercier, Hugo y Sperber, Dan. The enigma of reason. Cambridge, Massachusetts: Harvard University Press, 2017.

Nosofsky, Robert. "Attention, similarity, and the identification-categorization relationship." Journal of Experimental Psychology: General. 115(1) (1986): 39-57.

Peacocke, Christopher. A study of concepts. Cambridge, Massachusetts: M.I.T. Press, 1992.

Peacocke, Christopher. The realm of reason. Oxford: Oxford University Press, 2004.

Prinz, Jesse. Furnishing the mind. Cambridge, Massachusetts: M.I.T. Press, 2002.

Shecaira, Fábio. "Analogical arguments in ethics and law: a defense of a deductivist analysis." Informal Logic. 33(3) (2013): 406-437.

Smith, Edward y Medin, Douglas. Categories and concepts. Cambridge, Massachusetts: Harvard University Press, 1981.

Van Eemeren, Frans y Garssen, Bart. "Argumentation by analogy in stereotypical argumentative patterns." En Ribeiro, H. (ed.) Systematic approaches to argument by analogy (pp. 41-56). New York: Springer, 2014.

Van Eemeren, Frans y Snoek Henkemans, Francisca. Argumentation. Analysis and evaluation. New York: Routledge, 2017.

Waller, Bruce. “Classifying and analyzing analogies.” Informal logic. 21(3) (2001): 199-218.

Walton, Douglas. Methods of argumentation. Cambridge: Cambridge University Press, 2013.

Walton, Douglas, Reed, Christopher y Macagno, Fabrizio. Argumentation schemes. Cambridge: Cambridge University Press, 2008. 